\title{
Effect of Pulmonary Tuberculosis on Protein C, S, and Antithrombin-III among Therapy-naïve Ghanaian Adults; A Comparative Cross-Sectional Study
}

\section{Felix Osei-Boakye ( $\square$ foseiboakye1@gmail.com )}

Department of Medical Diagnostics, Faculty of Allied Health Sciences, Kwame Nkrumah University of Science and Technology, Kumasi, Ghana. https://orcid.org/0000-0001-5126-7424

\section{Otchere Addai-Mensah}

Department of Medical Diagnostics, Faculty of Allied Health Sciences, Kwame Nkrumah University of Science and Technology, Kumasi, Ghana.

\section{Michael Owusu}

Department of Medical Diagnostics, Faculty of Allied Health Sciences, Kwame Nkrumah University of Science and Technology, Kumasi, Ghana; Kumasi Centre for Collaborative Research in Tropical Medicine.

\section{Abdul-Razak Saasi}

Department of Medical Diagnostics, Faculty of Allied Health Sciences, Kwame Nkrumah University of Science and Technology, Kumasi, Ghana.

\section{Samuel Kwasi Appiah}

Department of Biomedical Laboratory Science, School of Allied Health Sciences, University for Development Studies, Tamale, Ghana.

\section{Charles Nkansah}

Department of Biomedical Laboratory Science, School of Allied Health Sciences, University for Development Studies, Tamale, Ghana.

\section{Yaw Amo Wiafe}

Department of Medical Diagnostics, Faculty of Allied Health Sciences, Kwame Nkrumah University of Science and Technology, Kumasi, Ghana.

\section{Alexander Yaw Debrah}

Department of Medical Diagnostics, Faculty of Allied Health Sciences, Kwame Nkrumah University of Science and Technology, Kumasi, Ghana; Kumasi Centre for Collaborative Research in Tropical Medicine.

\section{Research Article}

Keywords: Antithrombin-III, Haematological profile, Hypercoagulability, Protein C, Protein S, Pulmonary tuberculosis

Posted Date: December 30th, 2020 
DOI: https://doi.org/10.21203/rs.3.rs-135336/v1

License: (c) (1) This work is licensed under a Creative Commons Attribution 4.0 International License. Read Full License

Version of Record: A version of this preprint was published at Journal of Immunoassay and Immunochemistry on December 30th, 2020. See the published version at https://doi.org/10.1080/15321819.2021.2001002. 


\section{Abstract}

Background. Tuberculosis (TB) constitutes a global emergency as it affects one-third of the world's inhabitants. Although pulmonary Tuberculosis (PTB) is curable, immunological responses to the infection induce several haematological derangements. This study evaluated the effect of PTB on Protein C, Protein S, Antithrombin-III, and blood count parameters.

Methods. Ninety adults with ages $\geq 18$ years were purposively recruited: 60 PTB patients and 30 non-TB controls. All patients were diagnosed with sputum GeneXpert MTB/Rif assay. Blood specimens were collected from each participant for Protein C, S, Antithrombin-III and complete blood count.

Results. Pulmonary TB was associated with significantly reduced Protein C activity (101.46 [87.61-128.3] vs 121.44 [99.50-149.8] IU/L, $p=0.038)$, RBC (3.88 \pm 0.91 vs $4.80 \pm 0.55, p<0.0001), \mathrm{HgB}(10.24 \pm 2.42$ vs $11.78 \pm 1.42, p=0.0019), \mathrm{HCT}(32.21 \pm 7.79$ vs $42.05 \pm 4.97, p<0.0001), \operatorname{MCV}(83.80$ [79.33-90.08] vs 89.00 [83.75-92.00], $p=0.0133)$ and PDW (12.95 [10.73-15.00] vs 15.30 [14.18-15.93], $p<0.0001)$ compared to controls. Conversely, PTB patients were associated with significantly increased $\mathrm{MCH}(26.83 \pm 4.33 \mathrm{vs}$ $24.59 \pm 1.99, p=0.0086)$, TWBC (7.76 [6.06-9.78] vs 6.50 [4.85-7.50], $p=0.0047)$, Abs. GRAN (5.27 [3.306.71] vs 3.75 [2.48-4.75], $p=0.0226)$, RDW-CV (13.70 [13.20-15.43] vs 12.95 [12.50-13.65], $p<0.0001)$, MCHC (32.10 [28.70-35.63] vs 27.85 [27.40-28.53], $p<0.0001)$ and MPV (8.3 [6.7-9.7] vs 7.0 [6.4-7.5], $p=0.0027)$ compared to controls. The PTB patients were disproportionately affected with anaemia (91.7\%, $p=0.001)$, erythrocytopenia $(75.0 \% ; p \leq 0.001)$ and reduced HCT $(80.0 \%, p \leq 0.001)$. The frequency of thrombocytosis, leucocytosis, and granulocytosis $(50.0 \%, p=0.013 ; 23.3 \%, p=0.013 ; 18.3 \%, p=0.025$; respectively) in PTB patients were significantly higher than in controls.

Conclusion. Our findings suggest that PTB predisposes patients to hypercoagulability, with a significant reduction in Protein $\mathrm{C}$ activity but not Protein $\mathrm{S}$ and antithrombin-III. The condition causes derangements in erythrocytes, leucocytes, and thrombocytes, and disproportionately causes anaemia. Protein $\mathrm{C}$ activity and complete blood count are useful in the management of PTB and should be included in the routine workup for patients.

\section{Introduction}

Tuberculosis (TB) is a chronic and infectious disease [1] caused by a group of bacteria that belong to the Mycobacterium tuberculosis complex (MTBC) [2,3]. Mycobacterium tuberculosis is the preeminent species of the MTBC known to cause TB among humans [4]. In humans, TB disproportionately $(70 \%)$ infects the lungs resulting in pulmonary tuberculosis (PTB) [5], and is acquired when aerosols containing tubercle bacilli from an infected person are inhaled by a susceptible host [6]. Worldwide, the estimated tuberculosis burden is 1.86 billion cases, which approximates to $32 \%$ of the global population, with nearly nine million new infections recorded each year [7]. Tuberculosis burden in Africa is second after Asia and accounts for about $26 \%$ of the world's cases [8]. Also, it is reported to be the second most significant course of death in countries with poor and low economies [9] like Ghana. Such countries are known to 
harbour the greatest burden of cases and deaths (about 95\% and 98\%, respectively) associated with TB [10]. In Ghana, TB infects approximately 46,000 people every year [11] and more than $50 \%$ of them are adults [12].

Pulmonary TB induces haemostatic changes which are ascribed to the upregulation of procoagulant factors, diminished levels of anticoagulants and fibrinolytic factors [13]. Consequently, these altered processes culminate in increased coagulability [14] which may, in turn, lead to the formation of clots in deep veins (DVT) and obstruction of the blood flow in blood vessels [15]. A blood clot in deep veins is more frequent in older adults, and its rate of occurrence has a positive correlation with age [16]. It is estimated that $1.5 \%$ to $3.4 \%$ of TB infected patients suffer from such vascular complications [17] and could even exceed $10 \%$ since the exact incidence is not known [18].

Although some studies have explored haematological derangements among PTB patients in other parts of the world, most of these studies only highlighted derangements in blood clotting profiles and or platelet count, while they failed to include tests to measure activities of natural anticoagulants like protein C, S, and antithrombin-III and also, the full complement of the complete blood count (CBC) parameters. For instance, studies by Eteudo et al. [13] and Kutiyal et al. [19] assessed coagulation profiles but failed to measure anticoagulant activities. In Ethiopia, Kassa et al. [20] and Kahase et al. [21] measured only blood count parameters. Despite the thrombotic complications associated with PTB management of patients with the disease in Ghana does not include haematological assays to assess protein $\mathrm{C}, \mathrm{S}$ and antithrombin-III activities, and CBC which could provide information on their risk of thrombosis and associated sequelae. Thus, understanding the effect of PTB infection on natural anticoagulant activities among Ghanaian adults remains elusive.

This study, therefore, sought to determine the effect of PTB on protein C, S, and Antithrombin-III activities and $\mathrm{CBC}$ parameters among Ghanaian adults. This was to determine the usefulness of natural anticoagulant activities and CBC in the management of PTB.

\section{Materials And Methods}

\section{Study design/Study site}

This was a hospital-based comparative cross-sectional study conducted at the Nkawie-Toase Government Hospital, Ghana between November 2019 and May 2020. The hospital is among a selected few within Ashanti Region that operates a GeneXpert molecular-based rapid diagnostic analyser and has been designated a tuberculosis diagnostic referral centre by the National Tuberculosis Control Programme (NTP), Ghana. In addition to analysing sputum specimens collected at the hospital, the hospital's laboratory is also responsible for diagnosing TB in sputum specimens received from within the Atwima-Nwabiagya municipality as well as those referred from adjoining districts. The municipality is located on coordinates $6^{\circ} 40^{\prime} \mathrm{N} 1^{\circ} 49^{\prime} \mathrm{W}$. 


\section{Study population}

Ninety (90) participants with ages $\geq 18$ years were enrolled in the study using purposive sampling. This comprised 60 cases selected from newly diagnosed pulmonary TB patients who were naïve to antituberculosis therapy and 30 healthy control subjects. Each participant was assessed for eligibility using a pre-tested closed-ended questionnaire. Among information captured with the questionnaire were sociodemographic data such as gender, age, residence, education, occupation, and history of smoking and alcohol intake. Diagnosis of all cases was done using chest radiograph and participants whose results suggested an abnormality were made to produce sputum for confirmation and semi-quantification with GeneXpert molecular-based assay (MTB/Rif assay). Individuals with malaria, HIV, viral hepatitis, coagulopathies, and history of contraceptives and/or anti-inflammatory drug usage were excluded.

\section{Sputum analysis}

Freshly expectorated sputum specimen was diluted three folds ( 1 in 3 dilutions) by adding two volumes of the sample reagent to one volume of sputum. The sputum-sample reagent mixture was vigorously shaken for 10 seconds using the Vortex-Genie 2 mixer (Scientific Industries Inc., U.S.A) and incubated at room temperature for 10 minutes. The mixture was shaken a second time and further incubated for an additional 5 minutes. Once liquefaction of the sputum was complete the Xpert MTB/Rif cartridge was labelled and carefully opened without touching the rear side. A plastic bulb pipette was used to transfer $2 \mathrm{ml}$ of the liquefied sputum into the specimen chamber of the cartridge while avoiding the formation of air bubbles. The cartridge was then placed in and analysed with a four-channel GeneXpert rapid point of care molecular-based analyser (Cepheid, Sunnyvale, CA, U.S.A).

\section{Blood specimen collection and analysis}

Approximately 4.0 millilitres $(\mathrm{mL})$ of whole blood was collected aseptically by venipuncture from each participant using evacuated blood collection tubes containing 3.8\% sodium citrate and tri-potassium ethylenediaminetetraacetic acid ( $\mathrm{K}_{3}$ EDTA) anticoagulant (BD, Becton, Dickinson and Company, USA). The blood-containing tubes were gently inverted about 8 times to allow adequate mixing of anticoagulants and blood. The $\mathrm{K}_{3}$ EDTA blood was immediately used to measured complete blood count using the Horiba ABX Micros ES 60 automated haematological analyser (Horiba ABX, France). Citrated blood was centrifuged at 3000 revolutions per minute (rpm) for 15 minutes, and the plasma used to measure protein C, S, and antithrombin-III activities by solid-phase sandwich enzyme-linked immunosorbent assay (Melson, Melson Medical Corporation, China).

\section{Statistical data analysis}

The data was inputted into Microsoft Excel 2016 and transferred into IBM SPSS Statistics 23 for recoding and analysis. Visualization of the data was performed using GraphPad Prism 8.4.3. All data except for 
age, red blood cell, haemoglobin, haematocrit, mean cell haemoglobin, and absolute lymphocyte count were significantly skewed and non-parametrically distributed $(p \leq 0.05)$. The non-parametric data were presented as median and interquartile ranges (IQR), and parametric as mean and standard deviation (SD). Means of PTB and controls were compared using Levene's test for equality of variance and t-test for two independent groups. The Mann-Whitney's U test was used to compare non-parametric distributions between PTB cases and controls. Chi-square and Fisher's Exact tests were used appropriately to test association and differences in proportions between categorical data.

\section{Ethical consideration}

The study was approved by the Committee on Human Research, Publication and Ethics (CHRPE) of the School of Medicine and Dentistry at the Kwame Nkrumah University of Science and Technology, Kumasi (reference number: CHRPE/AP/245/20). Also, written permission was obtained from the management of Nkawie-Toase Government Hospital, and all participants consented by signing or thumb printing a consent form before enrolment.

\section{Results}

\section{Sociodemographic characteristics of the participants}

Table 1 shows frequency and differences in proportions of socio-demographic variables stratified by controls and pulmonary TB cases.

The overall mean age of the participants was $44.9 \pm 15.14$ years, with mean age significantly higher among the PTB patients than controls ( $48.88 \pm 13.66$ vs $36.93 \pm 15.01$ years; $p \leq 0.001)$. Of the total 90 participants involved in the study, majority $59(65.6 \%)$ were males, $37(41.1 \%)$ were between 38-57-year age bracket, 40 (44.4\%) were junior high school graduates, 72 (80.0\%) were informal sector workers, 18 $(20.0 \%)$ and $37(41.1 \%)$ had a history of smoking and alcohol intake, respectively.

Of the 60 PTB patients, 30 (50.0\%) were within 38-57 years, while 15 (25.0\%) each was recorded for age groups 18-37 years and $\geq 58$ years. Pulmonary TB was more prevalent in 38-57-year group (30 [50.0\%], $p=0.002)$, males (46 [76.7\%], $p=0.002)$, junior high school graduates (31 [51.7\%], $p=0.029)$, smokers (16 [26.7\%], $p=0.027$ ), and alcoholics (32 [53.3\%], $p=0.001)$. The prevalence of pulmonary TB did not significantly differ across residence and occupation (Table 1 ).

Table 1: Sociodemographic characteristics of the participants at the Nkawie-Toase Government Hospital, Ghana stratified by PTB cases and controls. 


\begin{tabular}{|c|c|c|c|c|}
\hline Variables & Total $(n=90)$ & Control $(n=30)$ & $\overline{\mathrm{PTB}}(n=60)$ & $p$-value \\
\hline Age (years) & $44.9 \pm 15.14$ & $36.93 \pm 15.01$ & $48.88 \pm 13.66$ & $\leq 0.001$ \\
\hline Age group & & & & 0.002 \\
\hline $\begin{array}{l}18-37 \\
38-57 \\
>58\end{array}$ & $\begin{array}{l}34(37.8) \\
37(41.1) \\
19(21.1\end{array}$ & $\begin{array}{l}19(63.3) \\
7(23.3) \\
4(13.3)\end{array}$ & $\begin{array}{l}15(25.0) \\
30(50.0) \\
15(25.0)\end{array}$ & \multirow{4}{*}{0.002} \\
\hline Gender & & & & \\
\hline Male & 59 (65.6) & 13 (43.3) & $46(76.7)$ & \\
\hline Female & $31(34.4)$ & $17(56.7)$ & $14(23.3)$ & \\
\hline Level of education & & & & \multirow[t]{6}{*}{0.029} \\
\hline No formal education & $12(13.3)$ & $7(23.3)$ & $5(8.3)$ & \\
\hline Primary school & $16(17.8)$ & $3(10.0)$ & $13(21.7)$ & \\
\hline Junior High & $40(44.4)$ & $9(30.0)$ & 31 (51.7) & \\
\hline Senior High & $16(17.8)$ & 7 (23.3) & $9(15.0)$ & \\
\hline Tertiary & $6(6.7)$ & $4(13.3)$ & $2(3.3)$ & \\
\hline Residence & & & & \multirow[t]{3}{*}{0.823} \\
\hline Rural & $45(50.0)$ & $16(53.3)$ & 29 (48.3) & \\
\hline Urban & $45(50.0)$ & $14(46.7)$ & $31(51.7)$ & \\
\hline Occupation & & & & \multirow[t]{4}{*}{0.148} \\
\hline Unemployed & $8(8.9)$ & $3(10.0)$ & $5(8.3)$ & \\
\hline Formal & $10(11.1)$ & $6(20.0)$ & $4(6.7)$ & \\
\hline Informal & $72(80.0)$ & $21(70.0)$ & $51(85.0)$ & \\
\hline History of smoking & & & & \multirow[t]{2}{*}{0.027} \\
\hline Yes & $18(20.0)$ & $2(6.7)$ & $16(26.7)$ & \\
\hline History of alcohol intake & & & & \multirow[t]{2}{*}{0.001} \\
\hline Yes & $37(41.1)$ & $5(16.7)$ & $32(53.3)$ & \\
\hline
\end{tabular}

Data are presented as frequencies with corresponding proportions in parentheses and Mean \pm Standard deviation; Pearson Chi-Square and Fisher's Exact tests were used to compare proportions between PTB and controls; Independent-Samples t-test was used to compare means between the groups; $p \leq 0.05$ was significant.

\section{Levels of protein C, S, and antithrombin-III activities of participants}

Figure 1 compares median activities of natural anticoagulant biomarkers (protein C, S and antithrombinIII) between PTB cases and controls. Protein $C$ activity was significantly reduced in the PTB group compared to controls (101.46 [87.61-128.3] vs 121.44 [99.50-149.8], $p=0.038)$, and the effect $(r)$ of PTB on protein $C$ activity was medium (0.3). However, protein $S$ activity (80.98 [66.66-101.3] vs 90.35 [67.45$110.6], p=0.280)$ and antithrombin-III activity (2.17 [1.97-2.96] vs 2.36 [2.10-2.87], $p=0.284)$ did not significantly differ between the PTB cases and controls (Figure 1).

\section{Haematological profile of participants}

Table 2 compares the means and medians respectively, of complete blood count parameters between PTB and control groups. Mean RBC ( $3.88 \pm 0.91$ vs $4.80 \pm 0.55 ; p<0.0001), \mathrm{HgB}(10.24 \pm 2.42$ vs $11.78 \pm 1.42$; $p=0.0019)$, and HCT $(32.21 \pm 7.79$ vs $42.05 \pm 4.97 ; p<0.0001)$ were significantly reduced in PTB compared 
to controls. Conversely, means of $\mathrm{MCH}(26.83 \pm 4.33$ vs $24.59 \pm 1.99 ; p=0.0086)$ and Abs. LYM $(2.24 \pm 1.06$ vs $1.87 \pm 0.71 ; p=0.0929)$ were increased in PTB than controls, however, only $\mathrm{MCH}$ showed a significant difference between PTB and controls.

Median TWBC (7.76 [6.06-9.78] vs 6.50 [4.85-7.50]; $p=0.0047)$, Abs. GRAN (5.27 [3.30-6.71] vs 3.75 [2.484.75]; $p=0.0226)$, RDW-CV (13.70 [13.20-15.43] vs 12.95 [12.50-13.65]; $p<0.0001), \mathrm{MCHC}(32.10$ [28.7035.63 ] vs 27.85 [27.40-28.53]; $p<0.0001)$, and MPV (8.3 [6.7-9.7] vs 7.0 [6.4-7.5]; $p=0.0027)$ were all significantly increased among PTB cases than controls. Median PLT (300.0 [115.3-495.5] vs 247.0 [195.5303.3]; $p=0.2721)$ and PCT $(0.222$ [0.106-0.288] vs 0.174 [0.144-0.211]; $p=0.1276)$ were increased in PTB, but did not significantly differ from that of the controls.

In contrast, median PDW (12.95 [10.73-15.00] vs 15.30 [14.18-15.93], $p<0.0001)$ and MCV (83.80 [79.3390.08 ] vs 89.00 [83.75-92.00], $p=0.0133$ ) were significantly reduced in PTB than controls. Also, Abs. MON was reduced in PTB than controls, but showed no significant difference between the groups (0.515 [0.400-0.908] vs 0.750 [0.475-1.000]; $p=0.2622$ ) (Table 2).

Table 2: Haematological profile of the study participants at Nkawie-Toase Government Hospital, Ghana stratified by PTB cases and controls.

\begin{tabular}{llll}
\hline Variables & Controls & PTB Cases & $p$-value \\
\hline RBC $\left(10^{6} / \mu \mathrm{L}\right)$ & $4.80 \pm 0.55$ & $3.88 \pm 0.91$ & $<0.0001$ \\
\hline $\mathrm{HgB}(\mathrm{g} / \mathrm{dL})$ & $11.79 \pm 1.42$ & $10.24 \pm 2.42$ & $\mathbf{0 . 0 0 1 9}$ \\
\hline $\mathrm{HCT}(\%)$ & $42.05 \pm 4.97$ & $32.21 \pm 7.79$ & $<\mathbf{0 . 0 0 0 1}$ \\
\hline $\mathrm{MCH}(\mathrm{Pg})$ & $24.59 \pm 1.99$ & $26.83 \pm 4.33$ & $\mathbf{0 . 0 0 8 6}$ \\
\hline Abs. Lym $\left(10^{3} / \mu \mathrm{L}\right)$ & $1.87 \pm 0.71$ & $2.24 \pm 1.06$ & 0.0929 \\
TWBC $\left(10^{3} / \mu \mathrm{L}\right)$ & $6.50(4.85-7.50)$ & $7.76(6.06-9.78)$ & $\mathbf{0 . 0 0 4 7}$ \\
\hline Abs. GRAN $\left(10^{3} / \mu \mathrm{L}\right)$ & $3.75(2.48-5.27)$ & $5.27(3.30-6.71)$ & $\mathbf{0 . 0 2 2 6}$ \\
\hline RDW-CV $(\%)$ & $12.95(12.50-13.65)$ & $13.70(13.20-15.43)$ & $<\mathbf{0 . 0 0 0 1}$ \\
\hline MCHC $(\mathrm{g} / \mathrm{dL})$ & $27.85(27.40-28.53)$ & $32.10(28.70-35.63)$ & $<\mathbf{0 . 0 0 0 1}$ \\
PLT $\left(10^{3} / \mu L\right)$ & $247.0(195.5-303.3)$ & $300(115.3-495.5)$ & 0.2721 \\
\hline MPV $(\mathrm{fL})$ & $7.0(6.4-7.5)$ & $8.3(6.7-9.7)$ & $\mathbf{0 . 0 0 2 7}$ \\
\hline PCT $(\%)$ & $0.174(0.144-0.211)$ & $0.222(0.106-0.288)$ & 0.1276 \\
\hline PDW $(\mathrm{fL})$ & $15.30(14.18-15.93)$ & $12.95(10.73-15.00)$ & $<\mathbf{0 . 0 0 0 1}$ \\
\hline MCV $(\mathrm{fL})$ & $89.00(83.75-92.00)$ & $83.80(79.33-90.08)$ & $\mathbf{0 . 0 1 3 3}$ \\
\hline Abs. MON $\left(10^{3} / \mu L\right)$ & $0.750(0.475-1.000)$ & $0.515(0.400-0.908)$ & 0.2622 \\
\hline
\end{tabular}

RBC: Red blood cell, HgB: Haemoglobin, HCT: Haematocrit, MCH: Mean cell haemoglobin, Abs. LYM: Absolute lymphocyte, TWBC: Total white blood cell, Abs. GRAN: Absolute granulocyte, RDW-CV: Red cell distribution width coefficient of variation, MCHC: Mean cell haemoglobin concentration, PLT: Platelet, MPV: Mean platelet volume, PCT: Plateletcrit, PDW: Platelet distribution width, MCV: Mean cell volume, Abs. MON: Absolute monocyte, PC: Protein C, PS: Protein S, AT-III: Antithrombin-III, PTB: Pulmonary tuberculosis, Independent samples t-test and Mann-Whitney's U test were used appropriately to compare parametric and non-parametric distributions respectively, between PTB cases and controls, $p$ was significant at $\leq 0.05$. 


\section{Frequency of haematological derangements in the study participants}

Figure 2 shows the frequency of various haematological derangements in the study participants stratified by PTB cases and controls. Increased percentage of the cases were significantly associated with leucocytosis (high TWBC) compared to controls (23.3\% [14/60] vs 3.3\% [1/30], $p=0.031$ ). Absolute counts of lymphocyte and monocyte were mostly normal and showed no significant difference between PTB and controls ( $p=0.351$ and $p=1.000$, respectively). Granulocytosis was the predominant granulocyte abnormality observed among the cases (18.3\% [11/60]), followed by granulocytopenia in $11.7 \%(7 / 60)$. Controls were mostly normal $93.3 \%$ (28/30) with only $6.7 \%(2 / 30)$ showing granulocytopenia. The difference in proportions between the groups was significant $(p=0.025)$.

A vast majority of the cases showed erythrocytopenia (75.0\% [45/60], $p \leq 0.001)$, anaemia $(91.7 \%[55 / 60]$, $p=0.001)$ and low HCT (80.0\% [48/60], $p \leq 0.001)$, and 35.0\% ([21/60], $p=0.004)$ had low MCV. Among the controls, 26 (86.7\%) showed low MCH compared to 34 (56.7\%) of the cases, while 7 (11.7\%) of cases had high $\mathrm{MCH}$ as against $0.0 \%$ in controls. One hundred per cent $(100 \%)$ of the controls showed low $\mathrm{MCHC}$, while among the cases $\mathrm{MCHC}$ was low in only 28 (46.7\%) and high in $13(21.7 \%)$. Pulmonary TB was significantly associated with $\mathrm{MCH}$ and $\mathrm{MCHC}(p=0.012$ and $p \leq 0.001$, respectively). Red cell distribution width was mostly normal, with $16.7 \%(10)$ and $3.3 \%(1)$ of the cases and controls respectively, showing high values. The difference, however, was not significant $(p=0.092)$.

Thrombocytosis was present in $50 \%$ (30/60) of the cases, whereas $30 \%(9 / 30)$ of the controls showed the derangement. Also, thrombocytopenia was present in $15.0 \%(9 / 60)$ and $3.3 \%(1 / 30)$ of cases and controls respectively; the association was significant $(p=0.013)$. Mean platelet volume was mostly normal, with $31.7 \%(19 / 60)$ and $48.7 \%(14 / 30)$ of the cases and controls respectively, showing low values. Low PCT was observed in $21.7 \%(13 / 60)$ and $6.7 \%(2 / 30)$ of cases and controls respectively, with only $1.7 \%$ showing high values. Pulmonary TB was significantly associated with PDW ( $p=0.005)$, but not MPV and PCT ( $p=0.317$ and $p=0.144$, respectively) (Figure 2).

\section{Discussion}

Tuberculosis (TB) remains the most frequent cause of mortality among adults presenting with transmissible diseases, and although treatment exists, affected individuals succumb to complications like thromboembolism and formation of clots in deep veins [22]. Consequently, we determined the effect of PTB on the level of activities of protein C (PC), protein S (PS), and antithrombin-III (AT-III) in therapynaive Ghanaian adults. Also, we reported the haematological profile in PTB patients. The cases were significantly older than controls with half of the infections within the 38-57-year group, which was consistent with findings of Wangdi et al. [23]. This association between old age and TB is ascribed to the vulnerability that comes with waning immunity in older people [24, 25]. Also, ageing causes immune depletion through the diminished function of the thymus and decreased propensity to synthesize 
cytokines and replace senescent $\mathrm{T}$ lymphocytes [25]. Also, more males were infected than females, and this increased susceptibility of the male gender to TB in the current study corroborates findings of Mukherjee et al., [26]. This is because, oestradiol in females induces a cellular immune response by stimulating interferon-gamma to activate macrophages in response to the infection $[27,28]$ whereas, testosterone which is higher in males, inhibits the body's immunity [28].

This study showed a significant reduction in the activity of PC but not PS and AT-III among PTB cases compared to controls, and the magnitude of the effect of PTB on PC activity was medium. This was consistent with findings of Turken et al. [29] and Kager et al. [15]. Marlar and Gausman [30] posit that although estimating the level of these biomarkers does not always suggest venous thromboembolic tendencies (phenotype), correctly determining the level of even a single biomarker in plasma is pathognomic of the gene expression (genotype). Our findings, therefore, affirms the early body of knowledge which suggests that PTB predisposes patients to increased coagulability and associated sequelae.

The study by Turken et al. [29] reported a significant decline in PC activity in PTB patients who were naïve to antituberculosis chemotherapy. Similarly, Kager et al. [15], reported significantly decreased concentrations of PC, antithrombin, and unbound PS in different groups of TB patients, and attributed these derangements to reduced anticoagulant processes that accompany TB infection. For instance, the reduction in PC activity observed in the present study is thought to result from negative regulation of the processes that culminate in PC synthesis which occur mainly via the inability to copy endothelial cell protein $\mathrm{C}$ receptor and thrombomodulin, thus, compromising the potential to activate PC [31]. Also, low production, high consumption and leakage that result from endothelial cell damage can cause a decline in PC levels [32].

Furthermore, Kager et al. [15] explained the anomaly in PS as been because, the majority of PS in the systemic circulation is bound to $\mathrm{C} 4 \mathrm{~b}$, a complement factor that increases greatly in TB and therefore the reduced concentration of unbound protein $S$ is justified to be the result of a rise in systemic $C 4 b$ that occurs in TB patients. Similar to PC, PS is a vitamin K-dependent protein synthesized primarily within liver cells [33,34], as a result, altered function of the liver will impair its production. Also, more than half (53.3\%) of our cases had recent or past history of alcoholism, a situation which can cause alcoholinduced liver inflammation and damage and consequently deficiency in protein S. This may have contributed to the low activity of protein S observed among PTB cases in this study.

Antithrombin, on the other hand, becomes used up and deactivated by inflammation-mediated degradation [31]. Also, immune mechanisms to inflammation cause the liberation of cells like neutrophils and associated cytokines to additionally diminish the activity of antithrombin [31].

The majority of blood count parameters investigated in this study were significantly deranged among PTB patients compared to controls. We observed significantly reduced RBC count, $\mathrm{HgB}$, and HCT among the PTB patients than controls, which corroborate findings of studies from Nigeria [35], Ethiopia [21] and India [36]. However, this was in contrast to findings from a longitudinal prospective study in Ethiopia by 
Kassa et al. [20] who reported rather high $\mathrm{HgB}$ and HCT values in treatment-naïve TB patients. The most plausible explanation is that Kassa et al. [20] included both pulmonary and extra-pulmonary TB patients and also compared values of untreated patients with those receiving treatment, but not with healthy controls as found in our study.

Anaemia has been identified as a frequent occurrence and known determinant for loss of lives in TB patients [36] and was by far the most distinctive haematological derangement observed among the patients in the present study. Approximately $92 \%$ (91.7\%) of the PTB patients were anaemic, $75.0 \%$ had erythrocytopenia and $80.0 \%$ had low red cell mass. These corroborate findings of Olaniyi et al. [37] and Padhariya and Shah [38] in which anaemia was observed among $90.3 \%$ and $88 \%$ of TB patients respectively. Also, the reduced $\mathrm{HgB}$ in PTB has been reported in other studies around the globe $[39,40]$. This PTB-associated anaemia is attributed to the involvement of cytokines, which interfere with the uptake, release and storage of iron culminating in a reduction in the amount of iron in the blood, and consequently reduces its accessibility to the erythrocytes for the production of haemoglobin $[20,35,41]$. During inflammation, there is an upsurge in the synthesis of cytokines like IFN-gamma, TNF-alpha, and interleukins (IL)-1, IL-6 [20] and IL-10 [42]. The interplay of these cytokines in the pathogenesis of anaemia in chronic disease like PTB has been widely reported in other studies. Interleukin 6 , liberated by cells of the innate immunity, induces the synthesis of hepcidin which hampers uptake of iron from the duodenum [42] and favours the synthesis of ferritin, and together these events retain more iron in storage than is required [41]. Furthermore, the upsurge in TNF-alpha and IL-1 directly inhibits renal synthesis and release of erythropoietin, which is a growth-stimulating hormone for the production of erythrocytes; production of erythroid progenitor cells in the bone marrow is thus inhibited leading to impaired synthesis of red cells and haemoglobin, with the net effect of anaemia [42].

Also, TNF-alpha and gamma-interferons induce the synthesis of unstable superoxide and nitric oxide species which can cause unmediated injury to progenitor erythroid cells [41, 43]. This hampers the expansion of progenitor population of the erythroid lineage and further result in abnormal synthesis and function of the red cells' stimulatory hormone (erythropoietin) which cause the bone marrow to react poorly [20] leading to reduced red cell mass, haemoglobin level and anaemia as the overall effect. Furthermore, the anaemia in TB has been linked to the longevity of Mycobacterium tuberculosis through its ability to directly metabolize heme to generate iron for its utilization and as a result causes a reduction in haemoglobin [21]. The erythrocytopenia and reduced red cell mass may also be ascribed to phagocytosis of red blood cells (erythrophagocytosis) which results in reduced survival of the cells and the inability for the bone marrow to replenish senescent cells due to the depleted serum iron [41].

In the initial phase of anaemia in chronic diseases like TB recycling of iron is modestly challenging and may manifest in most cases as moderate anaemia with normocytic erythrocytes, but as the disease progresses there is diminished uptake of iron from the intestines which results in a marked depletion of iron and a characteristic microcytosis of the erythrocytes [41]. This was evident in the present study by the significantly increased proportion of the cases that had microcytic erythrocytes, and also, increased variability in red cell size (RDW) which could be pathognomic of iron deficiency [20] among the TB cases. 
These findings were consistent with some recent studies [21, 35], but contrasting to findings of other studies from Nigeria by Akpan et al. [44] and Okafor et al. [45], plausibly because their studies recruited subjects with heterogeneous ages that encompassed both children and adults whereas we considered entirely adults.

The blood of PTB cases showed normal haemoglobinization (normochromasia) which was evident by the overall increased $\mathrm{MCH}$ and $\mathrm{MCHC}$ observed, and the increased proportion of the patients that recorded normal-to-high $\mathrm{MCH}$ and $\mathrm{MCHC}$ estimates. This corroborates a study by Kassa et al. [20], and was also consistent with a review study by Weiss and Goodnough [42], which posits that anaemia associated with chronic diseases presents with normocytic normochromic blood findings.

The nonspecific innate and cellular immunity remains the foremost preventive mechanism against the spread of M. tuberculosis [46]. This leads to the formation of a granuloma, the hallmark of the infection which causes an immune equilibrium between the bacillus and affected host [47]; it is characterised by increased leucocyte populations like macrophages and lymphocytes that encapsulate the microorganisms within the lungs. This natural response of leucocytosis explains the significantly increased TWBC count and the increased proportion of the cases that showed leucocytosis. Furthermore, as the disease becomes chronic, increased synthesis of cytokines by $T$ lymphocytes and macrophages occur leading to increased maturation of lymphocytes [41]. This was evident by the increased absolute lymphocyte count and the predominantly normal values observed among the cases, although not significant. However, significantly reduced absolute monocyte counts were observed among the cases, which may plausibly be the result of increased differentiation of monocytes to macrophages in response to the increased need to contain the tubercle bacilli and subsequently, their entrapment in granuloma shells. This may in turn lead to the formation of more monocyte-derived macrophages leading to reduced circulating monocytes. Neutrophils form the bulk of the granulocytes and are also usually the first cell type to arrive at the affected area [21]. This early immune response, therefore, justifies the significantly elevated granulocytes in PTB observed in this study.

Platelet count and PCT were increased among the PTB patients than controls although not significant, and was consistent with findings of Eteudo et al. [13]. Fifty per cent of the patients showed thrombocytosis $(p=0.013)$, which corroborate findings from other studies $[40,48,49]$. Most inflammatory diseases including PTB favour reactive thrombocytosis through acute phase response [48]. During this phase of the infection, IL- 6 and TNF-alpha are among several cytokines that are synthesized and released in large quantities, and these proteins induce the development of thrombocyte-producing precursors and consequently, increased synthesis of platelets in the blood [50]. However, Renshaw and Gould [51] posit that reactive thrombocytosis may not be apparent in all TB patients and that reporting on the increased thrombocyte count in TB patients is more relevant in already developed countries where exacerbation of the infection by other comorbidities is unlikely.

Mean platelet volume provides information on platelet size and function, and in most cases posit that bserved patients were unknown. has been useful in evaluating the reaction to therapy. Unlike PLT and 
PCT, MPV was significantly elevated in PTB patients and was consistent with findings of Tozkoparan et al. [39] who ascribed the derangement to elevated thrombopoietic activity. Conversely, PDW was significantly decreased in the cases $(p<0.0001)$ and the proportion of cases that had low PDW was significantly decreased than in the control group $(p=0.005)$. This suggests that there existed some form of platelet anisocytosis in the PTB population. Together, PDW, PCT, and MPV provide information on platelet activation/function; the elevated PDW, therefore, could represent changes in the surface area of the platelets following their activation. This is due to the immune involvement of platelets in active TB disease [52].

\section{Conclusions}

Our findings suggest that PTB predisposes patients to hypercoagulability, with a significant reduction in Protein $\mathrm{C}$ activity but not Protein $\mathrm{S}$ and antithrombin-III. The condition causes derangements in erythrocytes, leucocytes, and thrombocytes, and disproportionately causes anaemia which may culminate in hypoxia and subsequent death of patients. Protein $\mathrm{C}$ activity and complete blood count are useful in the management of PTB and should be included in the routine workup for patients.

\section{Declarations}

\section{Data Availability}

The datasets generated and or analyzed during the current study are available in the Harvard Dataverse repository, doi: 10.7910/DVN/6MMBSA.

\section{Conflicts of Interest}

The authors declare that there is no conflict of interest regarding the publication of this paper.

\section{Funding Statement}

The work did not receive any specific grant from funding agencies in public, commercial, or non-profitsectors.

\section{Supplementary Materials}

All relevant data are within the article.

\section{Acknowledgments}


We are grateful to Miss Barbara Aggrey and the entire staff of Nkawie-Toase Government Hospital Laboratory for their support. Also, we appreciate all participants for their patience and cooperation.

\section{References}

[1] X. Wang et al., "Spatiotemporal epidemiology of, and factors associated with, the tuberculosis prevalence in northern China, 2010-2014," BMC Infect. Dis., vol. 19, p. 365, 2019.

[2] A. Alelign, A. Zewude, T. Mohammed, S. Tolosa, G. Ameni, and B. Petros, "Molecular detection of Mycobacterium tuberculosis sensitivity to rifampicin and isoniazid in South Gondar Zone, northwest Ethiopia," BMC Infect. Dis., vol. 19, p. 343, 2019.

[3] J. Shi et al., "GeneXpert MTB/RIF Outperforms Mycobacterial Culture in Detecting Mycobacterium tuberculosis from Salivary Sputum,” Biomed Res. Int., vol. 2018, 2018.

[4] L. Jordao and O. V Vieira, "Tuberculosis: New Aspects of an Old Disease," Int. J. Cell Biol., vol. 2011, 2011.

[5] C. B. Duru, K. A. Uwakwe, K. C. Diwe, C. C. Nnebue, H. N. Chineke, and C. A. Emerole, "Prevalence of Active Pulmonary Tuberculosis among HIV Positive Patients Attending Adult HIV Clinic in a Teaching Hospital in Imo State, South East, Nigeria: A 6 Year Review (2006-2012)," Indian J. Med. Res. Pharm. Sci., vol. 1, no. 6, 2014.

[6] T. Ahmad, M. A. Jadon, M. Nasir, and M. N. K. Khattak, "Prevalence of sputum smear positive pulmonary tuberculosis at Dargai, District Malakand, Pakistan: A four year retrospective study," Egypt. J. Chest Dis. Tuberc., vol. 65, no. 2, pp. 461-464, 2016.

[7] N. S. Hochberg et al., "The complexity of diagnosing latent tuberculosis infection in older adults in long-term care facilities," Int. J. Infect. Dis., vol. 44, pp. 37-43, 2016.

[8] H. Kipruto et al., "The epidemiology of tuberculosis in Kenya, a high TB/HIV burden country (20002013)," Int. J. Public Heal. Epidemiol. Res., vol. 1, no. 1, pp. 002-013, 2015.

[9] D. Bikila et al., "Seasonality and Trend Analysis of Pulmonary Tuberculosis at St. Paul Hospital Millennium Medical," Austin J. Pulm. Respir. Med., vol. 4, no. 2, p. 1054, 2017.

[10] E. Ejeta, M. Legesse, and G. Ameni, "Preliminary Study on the Epidemiology of Tuberculosis in Nekemte and Its Surroundings -Western Ethiopia," Sci. Technol. Arts Res., vol. 1, no. 1, pp. 18-25, 2012.

[11] G. Aryee et al., "Estimating the incidence of tuberculosis cases reported at a tertiary hospital in Ghana: a time series model approach," BMC Public Health, vol. 18, p. 1292, 2018.

[12] M. P. Kwabla, D. K. Ameme, and P. Nortey, "Pulmonary Tuberculosis and Its Risk Factors among Inmates of a Ghanaian Prison," Int. J. Trop. Dis. Heal., vol. 9, no. 3, pp. 1-10, 2015. 
[13] A. N. Eteudo et al., "A Correlation between Tuberculosis Infection and Coagulation Parameters (In Mile Four Hospital, Abakaliki)," Ann. Adv. Med. Sci., vol. 1, no. 1, 2017.

[14] S. Janssen et al., "Hemostatic Changes Associated With Increased Mortality Rates in Hospitalized Patients With HIV-Associated Tuberculosis: A Prospective Cohort Study," J. Infect. Dis., vol. 215, pp. 247258, 2017.

[15] L. M. Kager et al., "Pulmonary tuberculosis induces a systemic hypercoagulable state," J. Infect., vol. 70, pp. 324-334, 2015.

[16] A. Gupta and R. Dixit, "Pulmonary Tuberculosis: A Neglected Risk Factor for Deep Venous Thrombosis," Int. J. Mycobacteriology, vol. 6, no. 2, pp. 184-6, 2017.

[17] A. Gupta, P. Mrigpuri, A. Faye, D. Bandyopadhyay, and R. Singla, "Pulmonary tuberculosis - An emerging risk factor for venous thromboembolism: A case series and review of literature," Lung India, vol. 34, no. 1, pp. 65-9, 2017.

[18] H. Kwas, S. Habibech, I. Zendah, and I. Elmjendel, "Pulmonary embolism and tuberculosis," Asian Cardiovasc. Thorac. Ann., vol. 22, no. 4, pp. 487-490, 2014.

[19] A. S. Kutiyal, N. Gupta, S. Garg, and H. S. Hira, "A Study of Haematological and Haemostasis Parameters and Hypercoagulable State in Tuberculosis Patients in Northern India and the Outcome with Anti-Tubercular Therapy," J. Clin. Diagnostic Res., vol. 11, no. 2, pp. OC09-0C13, 2017.

[20] E. Kassa, B. Enawgaw, A. Gelaw, and B. Gelaw, "Effect of anti-tuberculosis drugs on hematological profiles of tuberculosis patients attending at University of Gondar Hospital, Northwest Ethiopia," BMC Hematol., 2016.

[21] D. Kahase, A. Solomon, and M. Alemayehu, "Evaluation of Peripheral Blood Parameters of Pulmonary Tuberculosis Patients at St. Paul's Hospital Millennium Medical College, Addis Ababa, Ethiopia: Comparative Study," J. Blood Med., vol. 11, pp. 115-121, 2020.

[22] K. W. M. P. P. Kumarihamy, D. M. P. U. K. Ralapanawa, and W. A. T. A. Jayalath, "A rare complication of pulmonary tuberculosis: a case report," BMC Res. Notes, vol. 8, p. 39, 2015.

[23] K. Wangdi and M. R. Gurung, "The epidemiology of tuberculosis in Phuentsholing General Hospital: a six-year retrospective study," BMC Res. Notes, vol. 5, no. 1, p. 311, 2012.

[24] S.-H. Wang, B. Carruthers, and J. Turner, "The Influence of Increasing Age on Susceptibility of the Elderly to Tuberculosis," Open Longev. Sci., vol. 6, pp. 73-82, 2012.

[25] R. Byng-Maddick and M. Noursadeghi, "Does tuberculosis threaten our ageing populations?," $B M C$ Infect. Dis., vol. 16, p. 119, 2016. 
[26] A. Mukherjee, I. Saha, A. Sarkar, and R. Chowdhury, "Gender differences in notification rates, clinical forms and treatment outcome of tuberculosis patients under the RNTCP," Lung India, vol. 29, no. 2, pp. 120-122, 2012.

[27] P. Chinnakali, K. Selvaraj, P. Thekkur, G. Ramasamy, M. Thulasingam, and K. Vasudevan, "Age and Sex Differences in Sputum Smear Microscopy Results for Acid Fast Bacilli in a Tertiary Care Centre, South India," J. Respir. Med., vol. 2014, 2014.

[28] J. Feng et al., "Gender differences in treatment outcomes of tuberculosis patients in Taiwan: a prospective observational study," Clin. Microbiol. Infect., vol. 18, no. 9, pp. E331-E337, 2012.

[29] O. Turken et al., "Hemostatic changes in active pulmonary tuberculosis," Int. J. Tuberc. Lung Dis., vol. 6, no. 10, pp. 927-932, 2002.

[30] R. A. Marlar and J. N. Gausman, "Laboratory testing issues for protein C, protein S, and antithrombin," Int. J. Lab. Hematol., vol. 36, pp. 289-295, 2014.

[31] C. Teresa, K. A. Mathews, A. C. G. Abrams-Ogg, and D. Wood, "The Link Between Inflammation and Coagulation: Influence on the Interpretation of Diagnostic Laboratory Tests," Compend. Contin. Educ. Vet., pp. E1-E12, 2011.

[32] K. Koyama, S. Katayama, K. Tonai, J. Shima, T. Koinuma, and S. Nunomiya, "Biomarker profiles of coagulopathy and alveolar epithelial injury in acute respiratory distress syndrome with idiopathic/immune-related disease or common direct risk factors," Crit. Care, vol. 23, p. 283, 2019.

[33] B. Koscielniak, E. Wypasek, and A. Undas, "Determinants of Elevated Levels of Natural Anticoagulants in Healthy Subjects," Adv. Clin. Exp. Med., vol. 24, no. 5, pp. 791-800, 2015.

[34] M. C. Pintao et al., "Protein S levels and the risk of venous thrombosis: results from the MEGA casecontrol study," Blood, vol. 122, no. 18, pp. 3210-3219, 2019.

[35] E. I. Obeagu, I. L. Okoroiwu, H. U. Nwanjo, and D. C. Nwosu, "Evaluation of haematological parameters of tuberculosis patients in Umuahia," Eur. J. Pharm. Med. Res., vol. 6, no. 7, pp. 693-699, 2019.

[36] K. Rohini, M. S. Bhat, P. S. Srikumar, and A. M. Kumar, "Assessment of Hematological Parameters in Pulmonary Tuberculosis Patients," Indian J. Clin. Biochem., vol. 31, no. 3, pp. 332-335, 2016.

[37] J. A. Olaniyi, O. M. Ige, S. K. Rahamon, and O. G. Arinola, "Haematological Abnormalities in Treatment Naive Multidrug- Resistant Tuberculosis Patients with or Without Human Immunodeficiency Virus (HIV) Infection," Arch. Basic Appl. Med., vol. 4, pp. 57-62, 2016.

[38] J. D. Padhariya and S. N. Shah, "Haematological Profile of Patients of Pulmonary Tuberculosis," Ann. Pathol. Lab. Med., vol. 6, no. 10, 2019. 
[39] E. Tozkoparan, O. Deniz, E. Ucar, H. Bilgic, and K. Ekiz, "Changes in platelet count and indices in pulmonary tuberculosis," Clin. Chem. Lab. Med., vol. 45, no. 8, pp. 1009-1013, 2007.

[40] Ş. Füsun, E. Yazar, and P. Yıldız, "Prominent features of platelet count, plateletcrit, mean platelet volume and platelet distribution width in pulmonary tuberculosis," Multidiscip. Respir. Med., vol. 7, p. 38, 2012.

[41] M. F. Yacoub, H. F. Ferwiz, and F. Said, "Effect of Interleukin and Hepcidin in Anemia of Chronic Diseases," Anemia, vol. 2020, 2020.

[42] G. Weiss and L. T. Goodnough, "Anemia of Chronic Disease," N. Engl. J. Med., vol. 352, pp. 10111023, 2005.

[43] E. Poggiali, M. M. De Amicis, and I. Motta, "Anemia of chronic disease: A unique defect of iron recycling for many different chronic diseases," Eur. J. Intern. Med., vol. 25, no. 1, pp. 12-17, 2014.

[44] P. A. Akpan, J. O. Akpotuzor, and E. C. Akwiwu, "Some Haematological Parameters of Tuberculosis (TB) Infected Africans: The Nigerian Perspective," J. Nat. Sci. Res., vol. 2, no. 1, 2012.

[45] I. M. Okafor, A.-A. O. Eyo, E. A. Okon, and P. A. Akpan, "Some Haematological Parameters of Tuberculosis Infected Nigerians: A Study in Calabar - Nigeria," Glob. J. Pure Appl. Sci., vol. 19, pp. 95-100, 2013.

[46] S. Iqbal, U. Ahmed, and M. A. Khan, "Haematological parameters altered in tuberculosis," Pak J Physiol, vol. 11, no. 1, pp. 13-16, 2015.

[47] Q. Chai, Y. Zhang, and C. H. Liu, "Mycobacterium tuberculosis: An Adaptable Pathogen Associated With Multiple Human Diseases," Front. Cell. Infect. Microbiol., vol. 8, pp. 1-15, 2018.

[48] S. Rathod, D. R. Samel, P. Kshirsagar, and M. Pokar, "Thrombocytosis: can it be used as a marker for tuberculosis?," Int. J. Res. Med. Sci., vol. 5, no. 7, pp. 3082-3086, 2017.

[49] R. Kurup, K. Flemming, S. Daniram, S. Marks-James, and R. R. Martin, "Hematological and Biochemistry Profile and Risk Factors Associated with Pulmonary Tuberculosis Patients in Guyana," Tuberc. Res. Treat., vol. 2016, 2016.

[50] G. Gunluoglu, E. E. Yazar, N. S. Veske, E. C. Seyhan, and S. Altin, "Mean platelet volume as an inflammation marker in active pulmonary tuberculosis," Multidiscip. Respir. Med., vol. 9, p. 11, 2014.

[51] A. A. Renshaw and E. W. Gould, "Thrombocytosis Is Associated With Mycobacterium tuberculosis Infection and Positive Acid-Fast Stains in Granulomas," Am J Clin Pathol, vol. 139, pp. 584-586, 2013.

[52] S. H. Sadek, S. Farghaly, M. A. A. Abdou, and M. H. M. Abdel-Rahim, "Bedside inflammatory mediators in pulmonary tuberculosis," Egypt. J. Bronchol., vol. 11, pp. 260-267, 2017. 
Figures
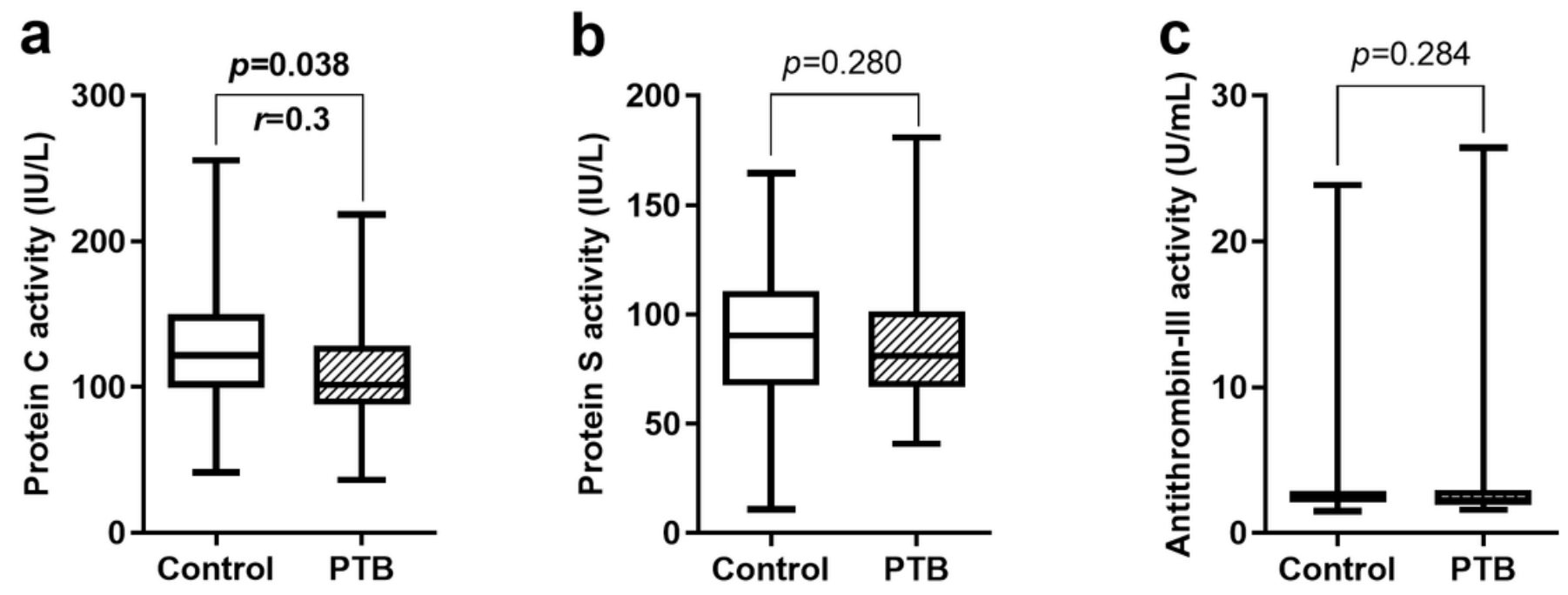

Figure 1

Levels of protein C, S, and antithrombin-III activities of participants at Nkawie-Toase Government Hospital, Ghana stratified by PTB cases and controls. Data are presented as box plots showing medians and interquartile ranges (IQR) of (a) Protein C activity, (b) Protein S activity and (c) Antithrombin-III activity; PTB= Pulmonary tuberculosis; $r=$ Effect size; Mann-Whitney nonparametric $U$ test was used to compare medians between PTB and controls; $p$ was significant at $\leq 0.05$. 

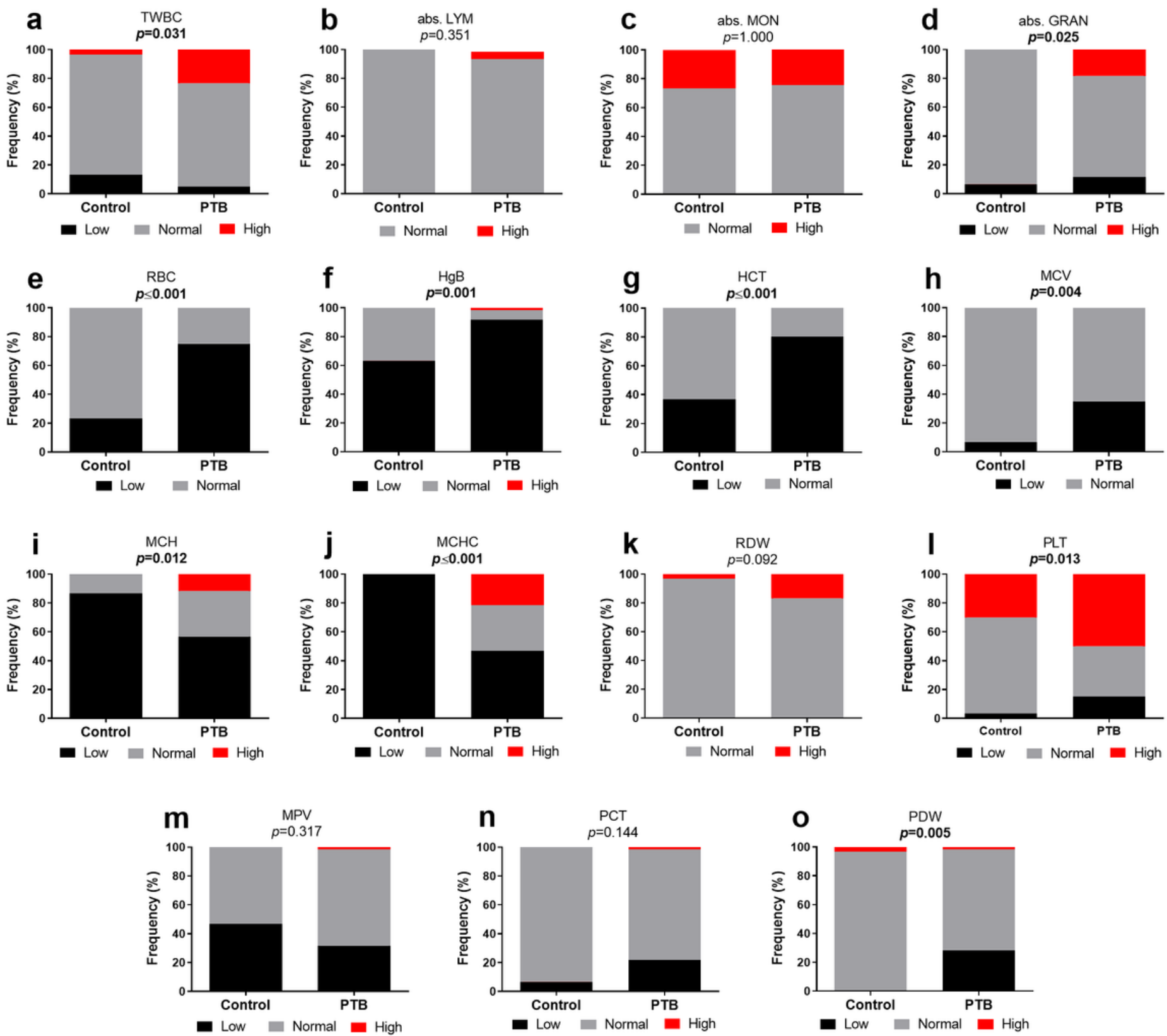

Figure 2

Frequency of haematological alterations in the study participants at Nkawie-Toase Government Hospital, Ghana stratified by PTB cases and controls Pearson Chi-Square and Fisher's Exact tests were used appropriately to compare difference in proportions between PTB and controls; (a) TWBC: Total white blood cell; (b) Abs. LYM: Absolute lymphocyte; (c) Abs. MON: Absolute monocyte; (d) Abs. GRAN: Absolute granulocyte; (e) RBC: Red blood cell; (f) HgB: Haemoglobin; (g) HCT: Haematocrit; (h) MCV: Mean cell volume; (i) MCH: Mean cell haemoglobin; (j) MCHC: Mean cell haemoglobin concentration; (k) RDW: Red cell distribution width; (I) PLT: Platelet; (m) MPV: Mean platelet volume; (n) PCT: Plateletcrit; (o) PDW: Platelet distribution width; PTB: Pulmonary tuberculosis; $\mathrm{p} \leq 0.05$ was significant. 